\title{
Decitabine enhances bortezomib treatment in RPMI 8226 multiple myeloma cells
}

\author{
YANG CAO, GUO-QIANG QIU, HAO-QING WU, ZHI-LIN WANG, \\ YAN LIN, WEI WU, XIAO-BAO XIE and WEI-YING GU \\ Department of Hematology, The First People's Hospital of Changzhou, Third Affiliated Hospital of Suzhou University, \\ Changzhou, Jiangsu 213003, P.R. China
}

Received July 19, 2015; Accepted June 24, 2016

DOI: $10.3892 / \mathrm{mmr} .2016 .5658$

\begin{abstract}
The present study investigated the interactions between decitabine (DAC) and bortezomib (BTZ) in RPMI 8226 multiple myeloma (MM) cells. Cells were exposed to DAC alone and in combination with BTZ for 48 h. A Cell Counting Kit- 8 assay was performed to assess the rate of proliferation inhibition in the cells. Cell apoptosis was investigated by Annexin V-fluorescein isothiocyanate and propidium iodide staining. Flow cytometry was used to detect the different cell cycle stages. Western blotting was performed to analyze the protein expression levels of poly(ADP-ribose) polymerase 1 (PARP-1), caspase-3, -9 and DNA (cytosine-5-)-methyltransferase 1 (DNMT1). Reverse transcription-quantitative polymerase chain reaction was used to assess DNMT1 gene expression. The combination of DAC and BTZ increased the proliferation inhibition, apoptotic rate and $\mathrm{G}_{0}-\mathrm{G}_{1}$ arrest compared with use of a single therapeutic agent. In addition, the combination treatment enhanced PARP-1 cleavage, caspase- 3 and caspase- 9 activation and downregulated the protein and mRNA expression levels of DNMT1. Therefore, the current study determined that the combination of BTZ and the epigenetic agent DAC may be a novel therapeutic strategy to improve the efficacy of BTZ in patients with MM.
\end{abstract}

Correspondence to: Professor Xiao-Bao Xie or Professor Wei-Ying $\mathrm{Gu}$, Department of Hematology, The First People's Hospital of Changzhou, Third Affiliated Hospital of Suzhou University, 185 Juqian Road, Changzhou, Jiansgu 213003 P.R. China

E-mail: czxxb1098@163.com

E-mail: guweiying2001@163.com

Abbreviations: AML, acute myeloid leukemia; BTZ, bortezomib; DAC, decitabine; DNMTs, DNA methyltransferase enzymes; HRP, horseradish peroxidase; MDS, myelodysplastic syndrome; MM, multiple myeloma; PARP, poly(ADP-ribose) polymerase; PBS, phosphate-buffered saline

Key words: decitabine, bortezomib, multiple myeloma, apoptosis

\section{Introduction}

Multiple myeloma (MM) is the second most common hematological malignancy characterized by the proliferation of clonal plasma cells in the bone marrow (1). The first-line treatment strategies for MM are combination regimens, including proteasome inhibitors and immunomodulatory agents. Bortezomib (BTZ) is the first proteasome inhibitor to be approved by the Food and Drug Administration (USA) for clinical use in patients with MM. The degradation of damaged or misfolded proteins is crucial in myeloma plasma cells, and BTZ treatment leads to a wide number of intracellular pleiotropic effects, including cell cycle arrest, induction of apoptosis and deregulation of nuclear factor- $\kappa \mathrm{B}(\mathrm{NF}-\kappa \mathrm{B})$ activity by preventing the normal breakdown of proteins in MM $(2,3)$. In addition, the incorporation of BTZ and novel immunomodulatory therapeutic agents, particularly thalidomide and lenalidomide, has markedly improved the rate of complete remission, progression-free survival, and overall survival in patients with MM $(4,5)$. However, despite available therapeutic strategies, MM remains incurable (6).

MM has a complex pathogenesis, which involves chromosomal instability (7), microRNA expression (8) and DNA methylation (9). DNA methylation is an epigenetic event that occurs at the cytosine residues in $\mathrm{CpG}$ dinucleotides and is catalyzed by DNA methyltransferase enzymes (DNMTs). DNA methylation is associated with gene silencing, which is important for the occurrence and development of MM (10). Therefore, it is possible that DNA methyltransferase inhibitors may be of benefit in the current clinical therapy of MM. Decitabine (5-aza-2'-deoxycytidin; DAC) is an epigenetic therapeutic agent that inhibits DNA methylation, which has been approved for the treatment of myelodysplastic syndrome and older patients with acute myeloid leukemia (AML) $(11,12)$. In addition to its use in hematological malignancy, DAC also exhibits antineoplastic effects against solid tumors (13-16). The molecular mechanism underlying its antitumor activity is that DAC induces DNMT inhibition, which contributes to DNA hypomethylation, gene activation, cell differentiation and apoptosis $(17,18)$.

Therapeutic agents used in combination often have synergistic anti-tumor effects and reduced side effects as lower concentrations of each individual agent are used. The present 
study investigated whether DAC may synergize with the frequently used anti-myeloma agent, BTZ. The results of the current study indicated that the anti-MM activity of the two agents was mutually reinforced, which resulted in an enhanced effect of proliferation inhibition, increased apoptosis and $\mathrm{G}_{0}-\mathrm{G}_{1}$ cell cycle arrest in the RPMI 8226 myeloma cell line. These events were associated with poly(ADP-ribose) polymerase 1 (PARP-1) cleavage, caspase-3 and-9 activation and DNA (cytosine-5-)-methyltransferase 1 (DNMT1) downregulation.

\section{Materials and methods}

Cell culture, proliferation inhibition assay and cell morphology analysis. RPMI 8226 cells (American Type Culture Collection, Manassas, VA, USA) were routinely cultured in RPMI-1640 media (Sigma-Aldrich, St. Louis, MO, USA), supplemented with $10 \%$ (v/v) heat-inactivated fetal bovine serum (GE Healthcare Life Sciences, Logan, UT, USA) in a humidified atmosphere with $5 \% \mathrm{CO}_{2}$ at $37^{\circ} \mathrm{C}$. Inhibition of cell proliferation was estimated using a Cell Counting Kit-8 (CCK-8; Dojindo Molecular Technologies, Inc., Kumamoto, Japan). Half inhibitory concentration $\left(\mathrm{IC}_{50}\right)$ of DAC in RPMI-8226 cells for $48 \mathrm{~h}$ was further calculated by a modified Kou-type method: $\lg \mathrm{IC}_{50}=\mathrm{Xm}-\mathrm{I}\left[\sum \mathrm{P}-(3-\mathrm{Pm}-\mathrm{Pn}) / 4\right]$, in which $\mathrm{Xm}, \lg$ maximum dose; I, $\lg$ (maximum dose/adjacent dose); $\Sigma \mathrm{P}$, sum of positive response rate; $\mathrm{Pm}$, the largest positive response rate; $\mathrm{Pn}$, the smallest positive response rate. Cells were observed and images were captured directly in flasks using a phase contrast microscope.

Chemicals and antibodies. BTZ was purchased from Millennium Pharmaceuticals, Inc. (Cambridge, MA, USA) and DAC was obtained from Sigma-Aldrich. These chemicals were dissolved in dimethyl sulfoxide (DMSO) and stored at $-20^{\circ} \mathrm{C}$. The primary antibodies used were as follows: Rabbit polyclonal anti-DNMT1 (cat. no. M0231L; New England Biolabs, Inc., Ipswich, MA, USA; 1:1,000); mouse monoclonal anti-PARP-1 (cat.no. sc-365315; Santa Cruz Biotechnology, Inc., Dallas, TX, USA; 1:1,000); rabbit polyclonal anti-caspase-9 (cat. no. 9502; $1: 1,000$ ), rabbit polyclonal anti-caspase-3 (cat. no. 9661; $1: 1,000$ ) and mouse monoclonal anti- $\beta$-actin (cat. no. 3700; 1:1,000) (Cell Signaling Technology, Danvers, MA, USA). The secondary antibodies used were as follows: Horseradish peroxidase (HRP)-conjugated goat anti-rabbit IgG (cat. no. 7074; 1:1,000) and HRP-conjugated horse anti-mouse IgG (cat. no. 7076; 1:1,000) (Cell Signaling Technology).

Cell apoptosis assays. Cell apoptosis was determined using a fluorescein isothiocyanate (FITC) Annexin V Apoptosis Detection kit (BD Biosciences, Franklin Lakes, NJ, USA) following the manufacturer's protocol. Following treatment with DAC and BTZ, RPMI 8226 MM cells were harvested $\left(2 \times 10^{5}\right)$, washed twice with ice-cold phosphate-buffered saline (PBS) and resuspended with binding buffer. Next, $5 \mu 1$ Annexin V-FITC was added to the suspended cells, mixed gently and incubated for $5 \mathrm{~min}$ in the dark. Following incubation, $5 \mu$ l propidium iodide (PI; Sigma-Aldrich) was added, gently mixed and incubated for a further $3 \mathrm{~min}$. Cells were analyzed using flow cytometry with CellQuest software version 5.1 (BD Biosciences).
Cell cycle analysis. Cells $\left(2 \times 10^{5}\right)$ were harvested, washed twice with ice-cold PBS and fixed with $75 \%$ cold ethanol at $4^{\circ} \mathrm{C}$ overnight. Then cells were incubated with RNase $(100 \mathrm{mg} / \mathrm{ml})$ for $30 \mathrm{~min}$ at $37^{\circ} \mathrm{C}$ and stained with PI $(250 \mathrm{mg} / \mathrm{ml})$ for another $30 \mathrm{~min}$ on ice and in the dark. The resulting cell suspension was subjected to flow cytometry. The fluorescent intensity of stained DNA was quantified using CellQuest software 5.1 (BD Biosciences).

Western blotting. Cells were harvested, washed with PBS and lysed with lysis buffer $(62.5 \mathrm{mM}$ Tris- $\mathrm{HCl}, \mathrm{pH} 6.8 ; 100 \mathrm{mM}$ DTT, $2 \%$ SDS and 10\% glycerol). Following incubation at $95^{\circ} \mathrm{C}$ for $10 \mathrm{~min}$, lysates were cooled on ice and centrifuged at $20,000 \mathrm{x} \mathrm{g}$ for $10 \mathrm{~min}$ at $4^{\circ} \mathrm{C}$. The Bradford method was used to quantify protein. Equal quantities $(10 \mu \mathrm{g})$ of protein extract were resolved by $6-15 \%$ sodium dodecyl sulfate-polyacrylamide gel electrophoresis and transferred to nitrocellulose membranes. Following blocking with 5\% non-fat milk in PBS, the membranes were probed with specific primary antibodies overnight at $4^{\circ} \mathrm{C}$. The membranes were washing in Tris Buffered Saline with Tween-20, and subsequently incubated with the HRP-conjugated secondary antibodies for $1 \mathrm{~h}$ at room temperature. The signals were detected by the chemiluminescence Phototope-HRP kit (EMD Millipore, Billerica, MA, USA), and imaged using a commercial imaging system (Fuji LAS400; Fuji Medical Systems, Stamford, CT, USA). The western blots were stripped using Restore Western Blot Stripping Buffer (Thermo Fisher Scientific, Inc., Waltham, MA, USA) and re-probed with anti- $\beta$-actin antibody as a loading control. All experiments were repeated three times and similar results were obtained.

$R N A$ extraction and reverse transcription-quantitative polymerase chain reaction ( $R T-q P C R)$. Total RNA was isolated using the TRIzol kit (Invitrogen; Thermo Fisher Scientific, Inc.) according to the manufacturer's protocols. The RNA was treated with DNase I (Promega Corporation, Madison, WI, USA). Complementary DNA was synthesized according to the manufacturer's protocol of the High-Capacity cDNA Reverse Transcription kit (Applied Biosystems; Thermo Fisher Scientific, Inc.). The analysis of DNMT1 and $\beta$-actin mRNA expression levels was performed by qPCR using SYBR Green PCR Master mix reagents (Applied Biosystems; Thermo Fisher Scientific, Inc.) on an Applied Biosystems 7300 Real Time PCR system (Thermo Fisher Scientific, Inc.). The specific primers used for detecting DNMT1 were as follows: Forward, 5'-GAT TTGTCCTTGGAGAACGGTG-3' and reverse, 5'-TGAGAT GTGATGGTGGTTTGCC-3'. The primers were synthesized by Sangon Biotech Co., Ltd. (Shanghai, China). Thermal cycler conditions were as follows: An initial incubation step of $95^{\circ} \mathrm{C}$ for $5 \mathrm{~min}$, followed by 50 cycles of $95^{\circ} \mathrm{C}$ for $15 \mathrm{sec}, 60^{\circ} \mathrm{C}$ for $15 \mathrm{sec}$ and $72^{\circ} \mathrm{C}$ for $45 \mathrm{sec}$, with a final $5 \mathrm{~min}$ extension step at $94^{\circ} \mathrm{C}$ to generate a melting curve. All experiments were performed in triplicate. Data were normalized to the housekeeping gene $\beta$-actin, and the relative abundance of transcripts was calculated using the comparative $2^{-\Delta \Delta \mathrm{Cq}}$ method (19).

Statistical analysis. Data are presented as the mean \pm standard deviation of three independent experiments. All statistical analyzes were performed using SPSS version 20 software 
A

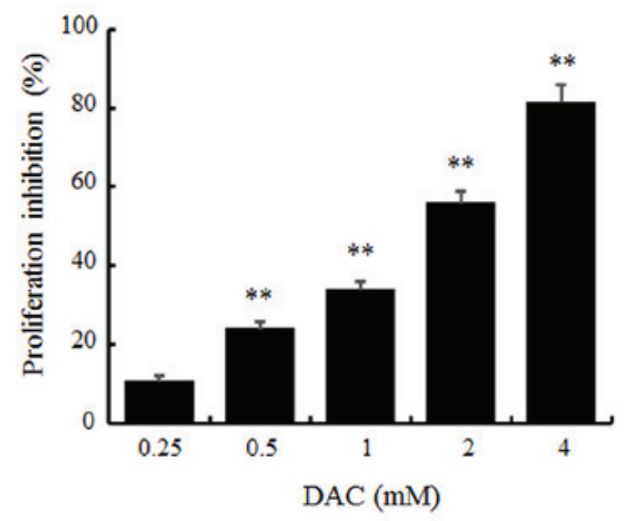

B

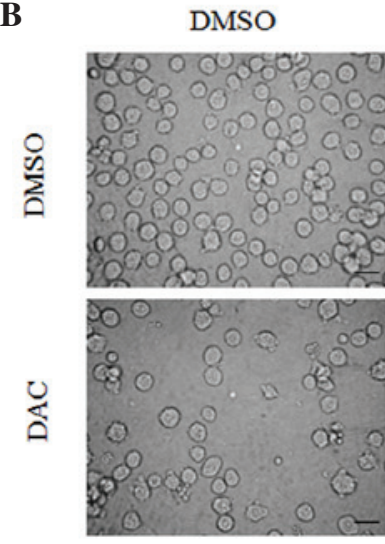

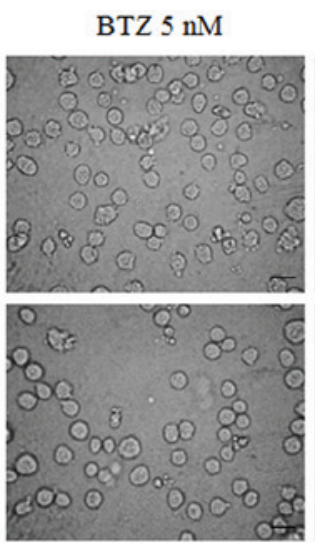
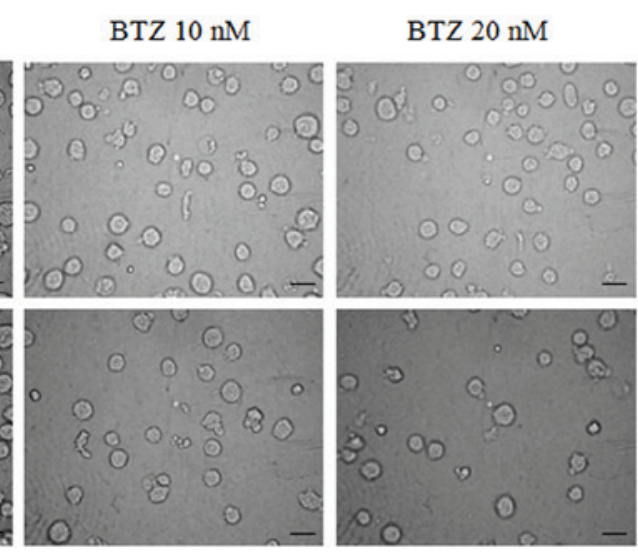

C

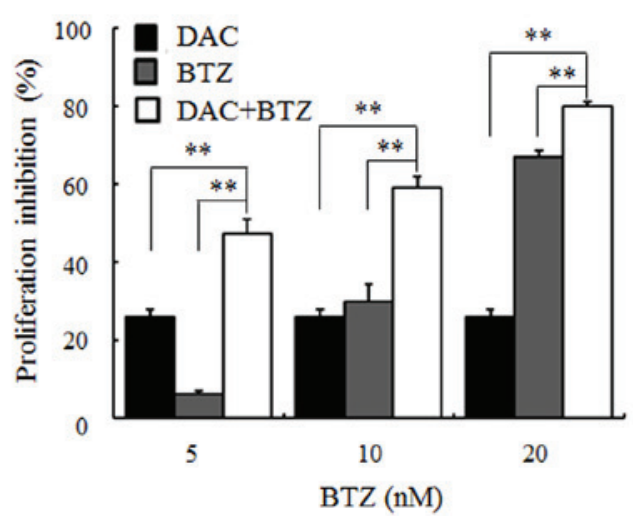

Figure 1. Inhibition of cell proliferation by DAC and BTZ in RPMI 8226 cells. (A) RPMI 8226 cells were exposed to the indicated concentrations of DAC for $48 \mathrm{~h}$ and growth inhibition was evaluated by Cell Counting Kit- 8 assay. ${ }^{* *} \mathrm{P}<0.01$ vs. the $0.25 \mathrm{mM}$ group. (B) Indicated concentrations of BTZ (5, 10, and $20 \mathrm{nM}$ ) and $0.5 \mathrm{mM}$ DAC were selected for the combined treatment. Cellular morphology was monitored using an inverted phase contrast microscope. Scale bar, $20 \mu \mathrm{m}$. (C) Quantification of proliferation inhibition of RPMI 8226 cells following treatment with DAC and BTZ. ${ }^{* *}$ P $<0.01$. Data are presented as the mean \pm standard deviation, from three independent experiments. DAC, decitabine; BTZ, bortezomib.

(IBM SPSS, Armonk, NY, USA). One-way analysis of variance and least significant difference tests were used to analyze the differences between groups. $\mathrm{P}<0.05$ was considered to indicate a statistically significant difference. Data are presented as the mean \pm standard deviation.

\section{Results}

Combination treatment with BTZ and DAC inhibits cell proliferation. A CCK-8 assay was performed to evaluate the effects of DAC on the proliferation of RPMI-8226 cells. As presented in Fig. 1A, exposure to increasing concentrations of DAC for $48 \mathrm{~h}$ inhibited cell proliferation in a dose-dependent manner. The inhibition rate was significantly increased to $10.69 \pm 0.41 \%, 26.04 \pm 0.91 \%, 33.80 \pm 2.09 \%, 55.79 \pm 1.04 \%$ and $81.37 \pm 2.36 \%$ with increasing doses of $0.25,0.5,1,2$ and $4 \mathrm{mM}$, respectively $(\mathrm{P}<0.01$; Fig. $1 \mathrm{~A})$. The half maximal inhibitory concentration $\left(\mathrm{IC}_{50}\right)$ of DAC for $48 \mathrm{~h}$ was $1.35 \mathrm{mM}$. To assess whether DAC enhanced the anti-MM effect of BTZ, the low dose $(0.5 \mathrm{mM})$ of DAC and different concentrations $(5,10$, and $20 \mathrm{nM}$ ) of BTZ were selected to treat myeloma cells. Inverted phase contrast microscopic examination demonstrated that, compared with the DMSO control group, cell density of the BTZ or DAC single agent groups was decreased and cellular debris was evident. This was also observed in the combined group (Fig. 1B). The CCK-8 assay was used to confirm the 
Table I. Cell cycle distribution of the different treatment groups was determined by flow cytometry analysis of DNA content.

\begin{tabular}{llll}
\hline Group & $\mathrm{G}_{0} / \mathrm{G}_{1}(\%)$ & $\mathrm{S}(\%)$ & $\mathrm{G}_{2} / \mathrm{M}(\%)$ \\
\hline DMSO & $29.32 \pm 2.32$ & $63.24 \pm 1.40$ & $7.45 \pm 0.94$ \\
DAC & $40.93 \pm 1.49^{\mathrm{a}}$ & $53.60 \pm 0.81^{\mathrm{a}}$ & $5.47 \pm 1.31$ \\
BTZ 10 nM & $40.96 \pm 0.88^{\mathrm{a}}$ & $54.20 \pm 1.57^{\mathrm{a}}$ & $4.82 \pm 0.94$ \\
DAC + BTZ 10 nM & $50.61 \pm 1.18^{\mathrm{b}}$ & $45.03 \pm 1.24^{\mathrm{b}}$ & $4.36 \pm 0.10$ \\
BTZ 20 nM & $49.64 \pm 0.92^{\mathrm{a}}$ & $45.31 \pm 1.41^{\mathrm{a}}$ & $5.05 \pm 1.19$ \\
DAC + BTZ 20 nM & $59.07 \pm 2.25^{\mathrm{b}}$ & $38.01 \pm 2.51^{\mathrm{b}}$ & $2.92 \pm 1.43^{\mathrm{b}}$
\end{tabular}

The data were expressed as the mean \pm standard deviation of three independent experiments. ${ }^{a} \mathrm{P}<0.05$ vs. control (DMSO); ${ }^{b} \mathrm{P}<0.05$ vs. either DAC or BTZ alone. DMSO, dimethyl sulfoxide; DAC, decitabine; BTZ, bortezomib.

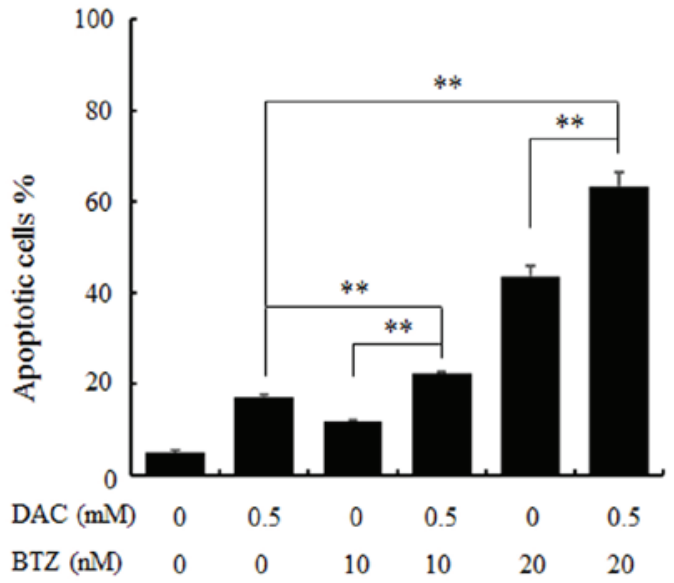

Figure 2. Combined treatment with DAC and BTZ increased the apoptotic rate of RPMI 8226 cells. RPMI 8226 cells were treated for $48 \mathrm{~h}$ and Annexin-V-positive cells were quantified by flow cytometry analysis. ${ }^{* *} \mathrm{P}<0.01$. Each experiment was performed in triplicate. DAC, decitabine; BTZ, bortezomib.

qualitative observations (Fig. 1C). Following treatment with 5,10 , and $20 \mathrm{nM} \mathrm{BTZ}$ for $48 \mathrm{~h}$, the inhibitory rate of cell growth was $6.21 \pm 0.75 \%, 29.80 \pm 4.48 \%$ and $67.15 \pm 1.51 \%$, respectively. When $0.5 \mathrm{mM}$ DAC was used in combination with the aforementioned concentrations of BTZ, the inhibitory rate was increased to $47.24 \pm 3.79 \%, 59.06 \pm 3.01 \%$ and $79.92 \pm 1.21 \%$. These results demonstrated that combined treatment with DAC and BTZ led to a significantly higher inhibition of cell growth compared with each agent alone ( $\mathrm{P}<0.01$; Fig. 1C).

DAC promotes BTZ-induced apoptosis of RPMI 8226 MM cells. To analyze the action between DAC and BTZ, the apoptotic rate of RPMI 8226 cells treated with these two therapeutic agents was examined by flow cytometry analysis using Annexin V and PI staining. As demonstrated in Fig. 2, the rates of Annexin V-positive cells in the BTZ groups (10 and $20 \mathrm{nM})$ were increased gradually with increasing dose, $11.82 \pm 0.31 \%$ and $43.69 \pm 4.21 \%$, respectively, compared with the control group, $4.86 \pm 0.71 \%(\mathrm{P}<0.01)$. In addition, in the combination treatment group $(0.5 \mathrm{mM}$ of DAC with each concentration of BTZ), a significant increase in the percentage of Annexin V positive cells was observed compared with the single treatment group ( $\mathrm{P}<0.01 ; 22.38 \pm 0.38 \%$ and $63.33 \pm 3.08 \%$, respectively). The results of apoptosis assay indicated that DAC may enhance the apoptotic rate of RPMI 8226 MM cells induced by BTZ.

DAC enhances BTZ-induced cell cycle arrest of RPMI 8226 MM cells. The effect of DAC and BTZ on cell cycle progression was further examined. As presented in Table I, the percentages of cells arrested at $\mathrm{G}_{0}-\mathrm{G}_{1}$ phase were $40.93 \pm 1.49,40.96 \pm 0.88$ and $49.64 \pm 0.92 \%$, following treatment with $0.5 \mathrm{mM}$ DAC alone, and 10 and $20 \mathrm{nM}$ BTZ alone, respectively. These results were significantly increased compared with the control $(\mathrm{P}<0.05$; $29.32 \pm 2.32 \%$ ). This indicates that DAC and BTZ single exposure induced high numbers of $\mathrm{G}_{0}-\mathrm{G}_{1}$ phase cells, with fewer $\mathrm{S}$ and $\mathrm{G}_{2} / \mathrm{M}$ phase cells. The combination treatment of DAC and 10 and $20 \mathrm{nM}$ BTZ resulted in a significantly increased number of cells in $\mathrm{G}_{0}-\mathrm{G}_{1}$ phase compared with the single treatment groups $(\mathrm{P}<0.01 ; 50.61 \pm 1.18 \%$ and $\mathrm{P}<0.01 ; 59.07 \pm 2.25 \%$, respectively). Overall, these findings suggested that DAC promoted the BTZ-induced cell cycle arrest of MM cells.

Combined treatment with BTZ and DAC leads to increased PARP-1 cleavage, caspase-3 and -9 activation. In order to determine the underlying mechanism of the increasing apoptotic rate induced by BTZ and DAC combination treatment, western blot analysis was performed. It was demonstrated that treatment with BTZ or DAC alone resulted in minimal effects, whereas combined treatment for up to $48 \mathrm{~h}$ resulted in an increase in caspase- 3 and caspase- 9 activation and enhanced PARP-1 cleavage (Fig. 3).

DNMT1 mRNA and protein expression levels are downregulated by combined treatment with DAC and BTZ. The clinical efficacy of DAC is due to its demethylating epigenetic action; therefore, combination treatment with DAC and BTZ may synergistically downregulate the mRNA and protein expression levels of DNMT1. RPMI 8226 MM cells were incubated with DAC and BTZ for $48 \mathrm{~h}$ and DNMT1 mRNA levels were determined using RT-qPCR (Fig. 4A). The single treatment with either BTZ or DAC alone was able to reduce DNMT1 mRNA expression to a certain extent; however, the combined treatment with the two agents resulted in a significant decrease in DNMT1 mRNA expression ( $\mathrm{P}<0.01$; Fig. 4A). This effect was also confirmed by the downregulation of DNMT1 protein expression levels analyzed by western blotting (Fig. 4B), the 


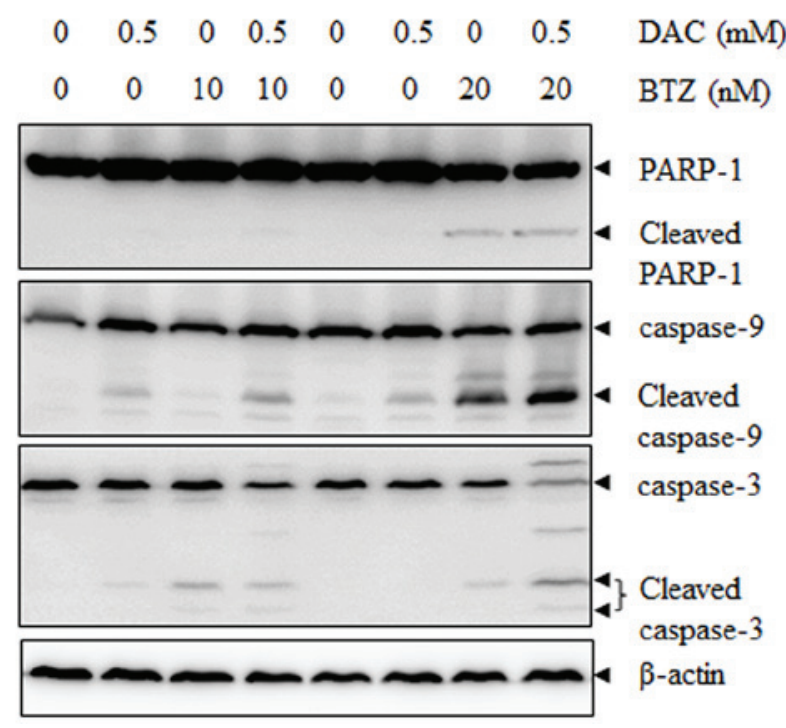

Figure 3. DAC and BTZ synergistically enhanced PARP-1 cleavage, along with caspase- 3 and -9 activation in RPMI 8226 cells. RPMI 8226 cells were exposed to DAC and/or BTZ for $48 \mathrm{~h}$. The indicated proteins were detected by western blotting with $\beta$-actin serving as a loading control. DAC, decitabine; BTZ, bortezomib; PARP-1, poly(ADP-ribose) polymerase 1.

A

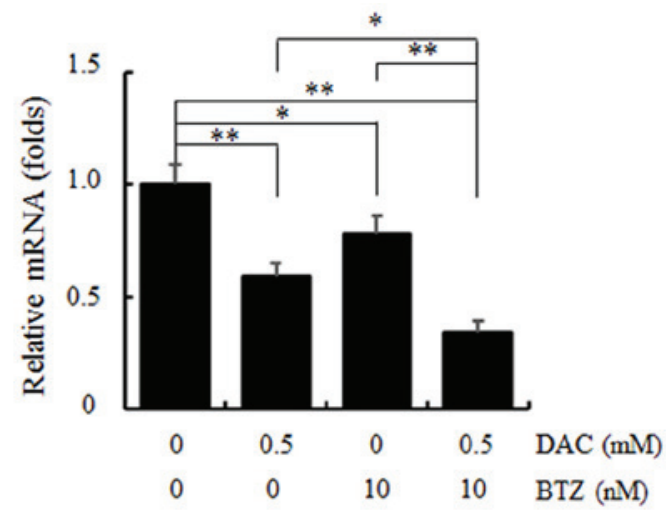

B

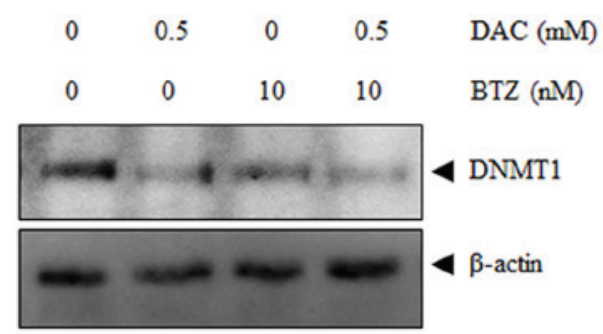

Figure 4. DAC and BTZ synergistically downregulated the mRNA and protein expression levels of DNMT1. (A) RPMI 8226 cells were treated with DAC and/or BTZ for $48 \mathrm{~h}$, reverse transcription-quantitative polymerase chain reaction was used to detect the DNMT1 mRNA level, expressed as the fold-change compared with the control level. Data are presented as the mean \pm standard deviation, from three independent experiments. ${ }^{*} \mathrm{P}<0.05$ and ${ }^{* *} \mathrm{P}<0.01$. (B) Western blotting was used to detect DNMT1 protein expression. $\beta$-actin served as a loading control. DAC, decitabine; BTZ, bortezomib; DNMT1, DNA (cytosine-5-)-methyltransferase 1.

results were consistent with the findings in previous studies in AML and MCL cells, which reported BTZ as a potent inhibitor of DNA methylation $(20,21)$.

\section{Discussion}

The present study investigated the combined effect of DAC and BTZ on the RPMI 8226 MM cell line and the underlying mechanism of action. It was determined that DAC and BTZ induce proliferation inhibition, apoptosis and $\mathrm{G}_{0}-\mathrm{G}_{1}$ arrest in RPMI 8226 cells. Notably, the combination treatment markedly enhanced these effects. DAC and BTZ used in combination also enhanced PARP-1 cleavage, caspase- 3 and -9 activation and downregulated the mRNA and protein expression levels of DNMT1.

MM remains an almost incurable disease, it is characterized by a high rate of relapse and drug resistance, despite the recent introduction of therapies, including proteasome inhibitors and immunomodulatory agents. Therefore, novel agents with improved efficacy and lower toxicities are required to develop the therapeutic strategies used in MM treatment. Epigenetic DNA methylation is involved in the pathogenesis and progression of MM (9), which has stimulated considerable interest in research on combinations of epigenetic agents with traditional chemotherapy. DAC and BTZ have long been approved for use in clinical treatment of specific diseases for, thus, their safety, efficacy, therapeutic window and side effects are well described. A previous study performed a phase 1 trial of BTZ and DAC in patients with poor-risk AML and has revealed their feasibility and preliminary clinical activity for use in AML treatment (22). However, little is known regarding their combined effects on MM. The present study determined, that DAC markedly enhanced the anti-MM effect of the frequently used proteasome inhibitor, BTZ. Therefore, a novel combined therapeutic strategy using BTZ and DAC may potentially enhance the effectiveness of BTZ in MM. However, this approach requires further investigation.

BTZ is a highly selective inhibitor of $26 \mathrm{~S}$ proteasome by reversibly binding to its active site. Proteasomes are protein complexes present in all eukaryotic cells located in the nucleus and the cytoplasm. The predominant function of a proteasome is to regulate the concentration of particular proteins and degrade unnecessary or damaged ones. This is important for the modulation of differentiation, apoptosis and cell cycle progression (23-26). Disorders in the proteasomal degradation system are involved in the pathogenesis of malignancy; therefore, protein degradation pathways are investigated as targets for cancer therapy, as confirmed by the successful clinical use of BTZ $(27,28)$. However, BTZ is not a perfect therapeutic agent. Previous studies indicated that BTZ has relatively narrow therapeutic window and may result in side effects, including cutaneous adverse reactions (29), neurotoxicity (30), and thrombocytopenia (31) during the treatment of MM. Therefore, the combination of BTZ with other novel therapeutic agents may alleviate its adverse effects by reducing the dosage used.

DNA methylation is an epigenetic regulation mechanism, which occurs at $\mathrm{CpG}$ islands. $\mathrm{CpG}$ islands frequently contain gene promoters or exons in normal mammalian cells. They are usually unmethylated, which allows transcriptional activity to control cell differentiation and phenotype (32). Methylation of $\mathrm{CpG}$ islands occurs at the carbon 5 position of the cytosine ring within $\mathrm{CpG}$ dinucleotides by a transfer of the methyl group from $S$-adenosyl-1-methionine (33). Various 
types of cancer often exhibit aberrant $\mathrm{CpG}$ methylation as this confers a growth advantage to neoplastic cells, which is associated with condensed chromatin, gene silencing and microRNA regulation. In MM, DNA methylation is important for the pathogenesis, progression and prognosis of the disease. Previous studies have suggested that numerous genes are hypermethylated in MM, including glutathione peroxidase 3, retinol binding protein 1 , secreted protein acidic and cysteine rich, transforming growth factor $\beta$ induced, cyclin-dependent kinase inhibitor $2 \mathrm{~A}$, suppressor of cytokine signaling 1 , fragile histidine triad 1 , death associated protein kinase 1 and transforming growth factor $\beta$ receptor 2 (34-38). Therefore, targeting DNA methylation may permit optimized therapy for MM, which warrants further investigation.

DNA methylation is catalyzed by DNMT1, 3a and $3 \mathrm{~b}$. DNMT3a and $3 \mathrm{~b}$ are important for de novo methylation, and DNMT1 is an important housekeeping gene for the maintenance of the established methylation pattern (39). Previous studies have determined that inhibition of DNMT1 by DAC induced DNA hypomethylation and reactivated silenced tumor-suppressor genes in leukemia cells and epithelial tumor cells $(40,41)$. The current study demonstrated that DAC and BTZ induced a significant decrease in DNMT1 expression levels, which may contribute to their antitumor activity. Information on the DNMT1 downregulation effect of BTZ in MM is scarce. The present study defined DNMT1 as a novel target, which mediated the BTZ antitumor effect. A significant reduction in the expression levels of DNMT1 was observed in MM cells following exposure to BTZ, this is consistent with the results reported in mantle cell lymphoma (MCL) and AML cells (20,21). In MCL models, BTZ has been reported as a potent inhibitor of DNA methylation, which decreased DNMT1 expression and induced global DNA hypomethylation (21). In AML models, BTZ was able to decrease Sp1 transcription factor (Sp1) protein levels and interfere with Sp1/NF- $\kappa$ B complex, which leads to DNMT1 downregulation and transcription of methylation-silenced genes (20). Overall, the present study supported BTZ as a novel and effective non-azanucleoside inhibitor of DNA methylation. In addition, as BTZ induced DNA hypomethylation with mechanisms different from those currently established hypomethylating azanucleosides, the combination of BTZ with azanucleosides, including DAC, may achieve synergistic anti-tumor activity with lower toxicity. However, the exact underlying mechanisms by which BTZ downregulates DNMT1 expression levels in MM require further investigation.

In conclusion, the results of the present study demonstrated that the epigenetic agent DAC promoted BTZ treatment in RPMI 8226 multiple myeloma cells. The combination of DAC and BTZ resulted in enhanced inhibition of proliferation, increased apoptosis and G0-G1 cell cycle arrest. Overall, these data supported BTZ as a novel and effective non-azanucleoside inhibitor of DNA methylation. In addition, as BTZ induced DNA hypomethylation with mechanisms different from those of currently established hypomethylating azanucleosides, the combination of BTZ with azanucleosides, including DAC, may achieve synergistic antitumor activity with lower toxicity. However, the exact underlying mechanisms by which BTZ downregulates DNMT1 expression levels in MM require further investigation.

\section{Acknowledgements}

The current study was supported in part by grants from the Funding of Science and Technology Bureau of Changzhou (grant no. CJ20130035) and Health and Research Funding of Changzhou (grant no. KY201442).

\section{References}

1. Palumbo A and Anderson K: Multiple myeloma. N Engl J Med 364: 1046-1060, 2011.

2. Chauhan D, Hideshima T, Mitsiades C, Richardson P and Anderson KC: Proteasome inhibitor therapy in multiple myeloma. Mol Cancer Ther 4: 686-692, 2005.

3. Hideshima T, Mitsiades C, Akiyama M, Hayashi T, Chauhan D, Richardson P, Schlossman R, Podar K, Munshi NC, Mitsiades N and Anderson KC: Molecular mechanisms mediating antimyeloma activity of proteasome inhibitor PS-341. Blood 101: 1530-1534, 2003.

4. Kumar SK, Rajkumar SV, Dispenzieri A, Lacy MQ, Hayman SR, Buadi FK, Zeldenrust SR, Dingli D, Russell SJ, Lust JA, et al: Improved survival in multiple myeloma and the impact of novel therapies. Blood 111: 2516-2520, 2008.

5. Moreau P, Attal M and Facon T: Frontline therapy of multiple myeloma. Blood 125: 3076-3084, 2015.

6. Ludwig H, Bolejack V, Crowley J, Bladé J, Miguel JS, Kyle RA, Rajkumar SV, Shimizu K, Turesson I, Westin J, et al: Survival and years of life lost in different age cohorts of patients with multiple myeloma. J Clin Oncol 28: 1599-1605, 2010.

7. Decaux O, Lodé L, Magrangeas F, Charbonnel C, Gouraud W, Jézéquel P, Attal M, Harousseau JL, Moreau P, Bataille R, et al: Prediction of survival in multiple myeloma based on gene expression profiles reveals cell cycle and chromosomal instability signatures in high-risk patients and hyperdiploid signatures in low-risk patients: A study of the Intergroupe Francophone du Myélome. J Clin Oncol 26: 4798-4805, 2008.

8. Gutiérrez NC, Sarasquete ME, Misiewicz-Krzeminska I, Delgado M, De Las Rivas J, Ticona FV, Fermiñán E, Martín-Jiménez P, Chillón C, Risueño A, et al: Deregulation of microRNA expression in the different genetic subtypes of multiple myeloma and correlation with gene expression profiling. Leukemia 24: 629-637, 2010.

9. Walker BA, Wardell CP, Chiecchio L, Smith EM, Boyd KD, Neri A, Davies FE, Ross FM and Morgan GJ: Aberrant global methylation patterns affect the molecular pathogenesis and prognosis of multiple myeloma. Blood 117: 553-562, 2011.

10. San-Miguel J, García-Sanz R and López-Pérez R: Analysis of methylation pattern in multiple myeloma. Acta Haematol 114 (Suppl 1): 23-26, 2005.

11. Curran MP: Decitabine: A review of its use in older patients with acute myeloid leukaemia. Drugs Aging 30: 447-458, 2013.

12. Jabbour E, Issa JP, Garcia-Manero G and Kantarjian H: Evolution of decitabine development: Accomplishments, ongoing investigations, and future strategies. Cancer 112: 2341-2351, 2008.

13. Nie J, Liu L, Li X and Han W: Decitabine, a new star in epigenetic therapy: The clinical application and biological mechanism in solid tumors. Cancer Lett 354: 12-20, 2014.

14. Primeau M, Gagnon J and Momparler RL: Synergistic antineoplastic action of DNA methylation inhibitor 5-AZA-2'-deoxycytidine and histone deacetylase inhibitor depsipeptide on human breast carcinoma cells. Int J Cancer 103: 177-184, 2003.

15. Numoto K, Yoshida A, Sugihara S, Kunisada T, Morimoto Y, Yoneda Y, Fujita Y, Nishida K, Ouchida M and Ozaki T: Frequent methylation of RASSF1A in synovial sarcoma and the anti-tumor effects of 5-aza-2'-deoxycytidine against synovial sarcoma cell lines. J Cancer Res Clin Oncol 136: 17-25, 2010.

16. Wang L, Amoozgar Z, Huang J, Saleh MH, Xing D, Orsulic S and Goldberg MS: Decitabine enhances lymphocyte migration and function and synergizes with CTLA-4 blockade in a murine ovarian cancer model. Cancer Immunol Res 3: 1030-1041, 2015.

17. Turcan S, Fabius AW, Borodovsky A, Pedraza A, Brennan C, Huse J, Viale A, Riggins GJ and Chan TA: Efficient induction of differentiation and growth inhibition in IDH1 mutant glioma cells by the DNMT inhibitor Decitabine. Oncotarget 4: 1729-1736, 2013. 
18. Ding L, Qiu L, Zhang J and Guo B: Camptothecin-induced cell proliferation inhibition and apoptosis enhanced by DNA methyltransferase inhibitor, 5-aza-2'-deoxycytidine. Biol Pharm Bull 32: 1105-1108, 2009.

19. Livak KJ and Schmittgen TD: Analysis of relative gene expression data using real-time quantitative PCR and the 2(-Delta Delta C(T)) method. Methods 25: 402-408, 2001.

20. Liu S, Liu Z, Xie Z, Pang J, Yu J, Lehmann E, Huynh L, Vukosavljevic T, Takeki M, Klisovic RB, et al: Bortezomib induces DNA hypomethylation and silenced gene transcription by interfering with Sp1/NF-kappaB-dependent DNA methyltransferase activity in acute myeloid leukemia. Blood 111: 2364-2373, 2008.

21. Leshchenko VV, Kuo PY, Jiang Z, Weniger MA, Overbey J, Dunleavy K, Wilson WH, Wiestner A and Parekh S: Harnessing Noxa demethylation to overcome Bortezomib resistance in mantle cell lymphoma. Oncotarget 6: 27332-27342, 2015

22. Blum W, Schwind S, Tarighat SS, Geyer S, Eisfeld AK, Whitman S, Walker A, Klisovic R, Byrd JC, Santhanam R, et al: Clinical and pharmacodynamic activity of bortezomib and decitabine in acute myeloid leukemia. Blood 119: 6025-6031, 2012.

23. Adams J: The proteasome: A suitable antineoplastic target. Nat Rev Cancer 4: 349-360, 2004.

24. Cecarini V, Bonfili L, Cuccioloni M, Mozzicafreddo M, Rossi G, Buizza L, Uberti D, Angeletti M and Eleuteri AM: Crosstalk between the ubiquitin-proteasome system and autophagy in a human cellular model of Alzheimer's disease. Biochim Biophys Acta 1822: 1741-1751, 2012

25. Bassermann F, Eichner R and Pagano M: The ubiquitin proteasome system-implications for cell cycle control and the targeted treatment of cancer. Biochim Biophys Acta 1843: $150-162,2014$

26. Neutzner A,LiS, Xu S and Karbowski M:The ubiquitin/proteasome system-dependent control of mitochondrial steps in apoptosis. Semin Cell Dev Biol 23: 499-508, 2012.

27. Yerlikaya A and Yöntem M: The significance of ubiquitin proteasome pathway in cancer development. Recent Pat Anticancer Drug Discov 8: 298-309, 2013.

28. Gatto S, Scappini B, Pham L, Onida F, Milella M, Ball G, Ricci C, Divoky V, Verstovsek S, Kantarjian HM, et al: The proteasome inhibitor PS-341 inhibits growth and induces apoptosis in $\mathrm{Bcr} / \mathrm{Abl}$-positive cell lines sensitive and resistant to imatinib mesylate. Haematologica 88: 853-863, 2003.

29. Patrizi A, Venturi M, Dika E, Maibach H, Tacchetti P and Brandi G: Cutaneous adverse reactions linked to targeted anticancer therapies bortezomib and lenalidomide for multiple myeloma: New drugs, old side effects. Cutan Ocul Toxicol 33 $1-6,2014$.
30. Cho Y, Hori M, Okoshi Y, Fujisawa F, Shinagawa A, Kudo D, Komeno T, Yoshida C, Katsura Y, Ota I, et al: Measurement of proteasome activity in peripheral blood mononuclear cells as an indicator of susceptibility to bortezomib-induced severe neurological adverse events in patients with multiple myeloma. Acta Haematol 134: 25-31, 2015.

31. Richardson PG, Sonneveld P, Schuster MW, Irwin D, Stadtmauer EA, Facon T, Harousseau JL, Ben-Yehuda D, Lonial S, San Miguel JF, et al: Safety and efficacy of bortezomib in high-risk and elderly patients with relapsed multiple myeloma. Br J Haematol 137: 429-435, 2007.

32. Li E and Zhang Y: DNA methylation in mammals. Cold Spring Harb Perspect Biol 6: a019133, 2014

33. Jones PA and Baylin SB: The fundamental role of epigenetic events in cancer. Nat Rev Genet 3: 415-428, 2002.

34. Kaiser MF, Johnson DC, Wu P, Walker BA, Brioli A, Mirabella F, Wardell CP, Melchor L, Davies FE and Morgan GJ: Global methylation analysis identifies prognostically important epigenetically inactivated tumor suppressor genes in multiple myeloma. Blood 122: 219-226, 2013

35. de Carvalho F, Colleoni GW, Almeida MS, Carvalho AL and Vettore AL: TGFbetaR2 aberrant methylation is a potential prognostic marker and therapeutic target in multiple myeloma. Int J Cancer 125: 1985-1991, 2009.

36. Galm O, Yoshikawa H, Esteller M, Osieka R and Herman JG: SOCS-1, a negative regulator of cytokine signaling, is frequently silenced by methylation in multiple myeloma. Blood 101: 2784-2788, 2003

37. Mateos MV, Garcia-Sanz R, López-Pérez R, Moro MJ, Ocio E, Hernández J, Megido M, Caballero MD, Fernández-Calvo J, Bárez A, et al: Methylation is an inactivating mechanism of the p16 gene in multiple myeloma associated with high plasma cell proliferation and short survival. Br J Haematol 118: 1034-1040, 2002.

38. Takada S, Morita K, Hayashi K, Matsushima T, Sawamura M, Murakami $\mathrm{H}$ and Nojima Y: Methylation status of fragile histidine triad (FHIT) gene and its clinical impact on prognosis of patients with multiple myeloma. Eur J Haematol 75: 505-510, 2005.

39. Herman JG and Baylin SB: Gene silencing in cancer in association with promoter hypermethylation. N Engl J Med 349: 2042-2054, 2003.

40. Robert MF, Morin S, Beaulieu N, Gauthier F, Chute IC, Barsalou A and MacLeod AR: DNMT1 is required to maintain $\mathrm{CpG}$ methylation and aberrant gene silencing in human cancer cells. Nat Genet 33: 61-65, 2003.

41. Tsai HC, Li H, Van Neste L, Cai Y, Robert C, Rassool FV, Shin JJ, Harbom KM, Beaty R, Pappou E, et al: Transient low doses of DNA-demethylating agents exert durable antitumor effects on hematological and epithelial tumor cells. Cancer Cell 21: 430-446, 2012. 